\title{
Etude analytique de la viscosité turbulente à l'aide du modèle K-L
}

\author{
Cristèle Chevalier $^{*}$; AndréTemperville ${ }^{* *}$
}

* Chargé de recherche, ORSTOM, LIA, 32 av. Varagnat, 93143 Bondy, France; tel :01 48027901

**Professeur Emérite,LEGI, BP 53, 38041 Grenoble Cedex 9, France; tel: 0476825068

Résumé

A partir d'un modèle de turbulence $K-L$, proposé par Lewellen (1977), nous déterminons une expression analytique de la longueur de mélange $L$. Cette expression valable sous $l^{\prime}$ hypothèse d'un gradient horizontal de pression nul, peut être appliquée dans la zone inertielle ou pour un écoulement du type de Couette. Nous montrons que cette expression, meilleure que l'expression linéaire classique, est en bon accord avec les calculs numériques. De plus les résultats obtenus permettent de construire une nouvelle solution pour le profil des vitesses qui peut être comparée avec la solution logarithmique classique.

\section{Abstract}

From the $K$ - $\ell$ model proposed by Lewellen (1977), an analytical expression of turbulence macroscale $L$ was established. This expression, obtained with the hypothesis of a null horizontal pressure gradient can be used for inertial or Couette flow. This formulation is better than the classical linear expression and in good agreement with numerical computations. We obtain a new solution for the velocity profil which can be compared with the logarithmic profil.

\section{Introduction}

La couche limite turbulente au voisinage d'un fond marin dans l'interaction houle-courant-sédiment a été largement étudiée au cours de cette dernière décennie, en particulier pour des fonds rugueux, en présence de sable. Les modèles ont souvent une fermeture turbulente de type $K-\varepsilon$ (A. Davies ( 1988 ) ou $K-L$, où $K$ représente l'énergie cinétique turbulente, $\varepsilon$ le taux de dissipation et $L$, la macro échelle des structures tourbillonnaires. Le modèle $K-L$ qui sera utilisé a été proposé, en 1977, par Lewellen(1977), puis appliqué au cas de la couche limite turbulente oscillatoire par P. Sheng et C. Villaret (1989) et généralisé, à l'étude de l'interaction houle et courant oblique par Huynh Thanh (1990). C. Villaret l'a aussi 
utilisé pour modéliser un écoulement de type Couette. En incluant les termes de gradient de densité le même système a été utilisé par Huynh Thanh (1994) et Guizien (1999) pour étudier la mise en suspension des sédiments. Le système d'équations à résoudre numériquement est décrit dans Huynh Tanh (1990).

Afin de pouvoir réduire les temps de calcul il est intéressant de pouvoir paramétrer certaines variables. Dans le cas d'un courant stationnaire, le système d'équations admet la solution élémentaire correspondant à $K=c s t e, L=\alpha z$ et donne un profil de vitesse logarithmique. Nous proposons de trouver une solution analytique plus réaliste vérifiant $d L / d z=0$ à la limite supérieure $z=h$ avec l'hypothèse de gradient de pression nul.

Cette solution valable dans la zone inertielle et pour un écoulement de type Couette, permettra d'une part de tester la modélisation numérique du système complet ( non linéaire ) et d'autre part de vérifier la sensibilité de certains paramètres. Dans le paragraphe 2 nous rappelons les équations générales. Une nouvelle solution est calculée pour un écoulement permanent dans le paragraphe 3 , et elle est comparée avec les solutions numériques dans le paragraphe 4 . Enfin dans paragraphe 5 nous étudions la nouvelle solution pour le profil des vitesses.

\section{Hypothèses et équations}

Nous considérons un écoulement, unidirectionnel en régime turbulent et sans stratification. Sous l'hypothèse de Boussinesq, l'équation de quantité de mouvement et les quatre équations pour les tensions de Reynolds $\left(\overline{u^{\prime} u^{\prime}}, \overline{v^{\prime} v^{\prime}}, \overline{w^{\prime} w^{\prime}}\right)$ et $\overline{u^{\prime} w^{\prime}}$ s'écrivent :

$$
\begin{aligned}
& \frac{\partial u}{\partial t}=-\frac{1}{\rho} \frac{\partial P}{\partial x}+\frac{\partial}{\partial z}\left(-\overline{u^{\prime} w^{\prime}} \frac{\partial u}{\partial x}\right) \\
& \frac{\partial \overline{u^{\prime 2}}}{\partial}=-2 \overline{u^{\prime} w^{\prime}} \frac{\partial u}{\partial z}-\frac{q}{L}\left[\overline{u^{\prime 2}}-\frac{q^{2}}{3}\right]-\frac{q^{3}}{12 L}+v_{c} \frac{\partial}{\partial z}\left(q L \frac{\partial \overline{u^{\prime 2}}}{\partial z}\right) \\
& \frac{\partial \overline{v^{\prime 2}}}{\partial}=-\frac{q}{L}\left[\overline{v^{\prime 2}}-\frac{q^{2}}{3}\right]-\frac{q^{3}}{12 L}+v_{c} \frac{\partial}{\partial z}\left(q L \frac{\left.\partial \frac{v^{\prime 2}}{\partial z}\right)}{\partial \overline{w^{\prime 2}}}=-\frac{q}{L}\left[\overline{w^{\prime 2}}-\frac{q^{2}}{3}\right]-\frac{q^{3}}{12 L}+v_{c} \frac{\partial}{\partial z}\left(q L \frac{\partial \frac{w^{\prime 2}}{\partial z}}{\partial z}\right)\right. \\
& \frac{\partial u^{\prime} w^{\prime}}{\partial \partial z}=-\frac{\partial w^{\prime 2}}{\partial z}-\frac{q}{L} \overline{u^{\prime} w^{\prime}}+v_{c} \frac{\partial}{\partial z}\left(q L \frac{\partial \frac{u^{\prime} w^{\prime}}{\partial z}}{\partial z}\right)
\end{aligned}
$$

Où $u^{\prime}, v^{\prime}, w^{\prime}$ représentent les fluctuations turbulentes par rapport aux vitesses moyennes $u, v, w ; q$ correspond à la vitesse turbulente; et $\mathrm{vc}=0.3$ d'après Lewellen (1977). 
Sachant que $q^{2}=2 K=\overline{u^{\prime} u^{\prime}}+\overline{v^{\prime} v^{\prime}}+\overline{w^{t} w^{\prime}}$, l'équation d'évolution de l'énergie cinétique turbulente $K$ s'obtient en sommant les équations $2,3,4$.

$\frac{\partial q^{2}}{\partial t}=2 v_{1}\left(\frac{\partial u}{\partial z}\right)^{2}-\frac{q^{3}}{4 L}+v_{c} \frac{\partial}{\partial z}\left(q L \frac{\partial q^{2}}{\partial z}\right)$

$v_{t}$ représente la viscosité turbulente définie par $\overline{u^{t} w^{\prime}}=-v_{t} \frac{\partial u}{\partial z}$;

Selon Lewellen (1977), L est modélisée par :

$\frac{\partial}{\partial z}=-S_{1} \frac{v_{t}}{q^{2}}\left(\frac{\partial u}{\partial z}\right)^{2} L+S_{2} q+v_{c} \frac{\partial}{\partial z}\left(q L \frac{\partial}{\partial z}\right)+\frac{S_{4}}{q}\left[\frac{\partial}{\partial z}(q L)\right]^{2}$

Avec $\mathrm{S} 1=0.35, \mathrm{~S} 2=0.075, \mathrm{~S} 4=-0.375$ et $\mathrm{vc}=0.3$.

Nous proposons de résoudre ce système avec l'hypothèse de gradient de pression nul $(\partial P / \partial z=0)$ et $\partial L / \partial z=0$ pour $z=h$.

\section{Résolution}

Pour un écoulement permanent les équations s'écrivent :

$$
\begin{aligned}
& 0=-\frac{1}{\rho} \frac{\partial p}{\partial x}+\frac{\partial}{\partial z}\left(-\overline{u^{\prime} w^{\prime}}\right) \\
& 0=-2 \overline{u^{\prime} w^{\prime}} \frac{\partial s}{\partial z}-\frac{q}{L}\left[\overline{u^{\prime 2}}-\frac{q^{2}}{3}\right]-\frac{q^{3}}{12 L}+v_{c} \frac{\partial}{\partial z}\left(q L \frac{\overline{\partial u^{\prime 2}}}{\partial z}\right) \\
& 0=-\frac{q}{L}\left[\overline{v^{\prime 2}}-\frac{q^{2}}{3}\right]-\frac{q^{3}}{12 L}+v_{c} \frac{\partial}{\partial z}\left(q L \frac{\partial^{\prime 2}}{\partial z}\right) \\
& 0=-\frac{q}{L}\left[\overline{w^{\prime 2}}-\frac{q^{2}}{3}\right]-\frac{q^{3}}{12 L}+v_{c} \frac{\partial}{\partial z}\left(q L \frac{\partial w^{\prime 2}}{\partial z}\right) \\
& 0=-\overline{w^{\prime 2}} \frac{\partial u}{\partial z}-\frac{q}{L} \overline{u^{\prime} w^{\prime}}+y_{c} \frac{\partial}{\partial z}\left(q L \frac{\partial w^{\prime}}{\partial z}\right) \\
& 0=2 v_{t}\left(\frac{\partial u}{\partial z}\right)^{2}-\frac{q^{3}}{4 L}+v_{c} \frac{\partial}{\partial z}\left(q L \frac{\partial q^{2}}{\partial z}\right) \\
& 0=-S_{1} \frac{v_{t}}{q^{2}}\left(\frac{\partial t}{\partial z}\right)^{2} L+S_{2} q+v_{c} \frac{\partial}{\partial z}\left(q L \frac{\partial L}{\partial z}\right)+\frac{S_{4}}{q}\left[\frac{\partial}{\partial z}(q L)\right]^{2}
\end{aligned}
$$

Avec l'hypothèse de gradient de pression nul $(\partial P / \partial z=0)$, nous déduisons, d'après l'équation 8 , que $-\overline{u^{\prime} w^{\prime}}=u^{*^{2}}=c s t e$. Sous l'hypothèse d'homogénéité locale de la turbulence qui permet de négliger les termes de diffusion, nous 
déduisons des équations $9,10,11$ que $\overline{u^{\prime} u^{\prime}}=2 \overline{v^{\prime} v^{\prime}}=2 \overline{w^{\prime} w^{\prime}}=K$, de l'équation 12 que $v_{t}=q L / 4$, et de l'équation 13 que $d u / d z=\sqrt{K} / L$. Ceci entraine alors que $-\overline{u^{\prime} w^{\prime}}=u^{* 2}=\sqrt{2} K / 4$ est constant. Cette solution, en accord parfait avec l'hypothèse précédente, est une solution exacte du système.

En tenant compte des résultats précédents et après simplifications, l'équation d'évolution de L (équations 14 ) s'écrit alors:

$$
\frac{S_{1} / 8-S_{2}}{v_{c}}=L \frac{d^{2} L}{d z^{2}}+\left(1+S_{4} / v_{c}\right)\left(\frac{d L}{d z}\right)^{2}
$$

En définissant $\alpha$ et $\gamma$ tels que :

$$
\left(v_{c}+S_{4}\right) \alpha^{2}=S_{\mathrm{i}} / 8-S_{2} \quad \text { et } \quad \gamma=-\left(S_{1} / 8-S_{2}\right) /\left(v_{c} \alpha^{2}\right)
$$

l'équation (15) s'écrit :

$$
L \frac{d^{2} L}{d z^{2}}=\gamma\left[\left(\frac{d L}{d z}\right)^{2}-\alpha^{2}\right]
$$

et en posant $t=L / \alpha \quad$ et $\quad t^{\prime}=d t / d z$, on obtient :

$$
t t^{\prime \prime}=\gamma\left(t^{2}-1\right)
$$

Une solution «évidente » de cette équation est la solution linéaire $L=\propto z$ qui correspond pour la vitesse u à la solution logarithmique classique. Cette solution ne vérifie pas la condition imposée à la limite supérieure $(\partial L / \partial z=0)$. II nous faut chercher une solution plus complexe.

Une première intégration de cette équation donne :

$$
t^{\prime 2}=1+\beta t^{2 \gamma}
$$

où $\beta$ est une constante d'intégration que l'on précisera par la suite.

Si l'on considère les valeurs de $S_{1}, S_{2}$ et $v_{c}$ proposées par Lewellen, $2 \gamma=0.5$ et l'équation 16 s'écrit:

$$
t^{\prime}=\sqrt{1+\beta \sqrt{t}}
$$

En posant $V=\sqrt{1+\beta \sqrt{t}}$ et compte tenu que $t=0$ et $V=1$ pour $z=0$ on obtient après intégration :

$$
z=\frac{4}{\beta^{2}} \frac{[V-1]^{2}[V+2]}{3}=\frac{4}{3 \beta^{2}}\left(V^{3}-3 V+2\right)
$$

La condition à la limite supérieure $t^{\prime}=V=0$ pour $z=h$ donne $\beta^{2}=8 / 3 h$ En posant $t^{\prime}=V=2 \cos (\theta)$ on en déduit l'expression pour $L$ :

$$
L=3 h \alpha\left(1-4 \cos ^{2} \theta\right)^{2} / 8 \quad \text { avec } \cos (3 \theta)=z / h-1 \quad \theta \in[\pi / 3, \pi / 2]
$$

Il est possible alors de calculer la viscosité turbulente $v_{t}$ et la vitesse u à l'aide du paramètre $\theta$. Une étude plus détaillée sera faite au paragraphe 5. 


\section{Comparaison avec des solutions numériques}

La valeur $\gamma=0,25$ utilisée correspond aux coefficients proposés par Lewellen. Afin de vérifier la sensibilité du système à ces coefficients, on fait varier la valeur de $\gamma$ au voisinage de 0,25 , et on résout numériquement l'équation 16 qui ne peut plus être résolue analytiquement. La valeur théorique de $t=L / \alpha$ est calculée d'après les formules précédentes obtenues avec $2 \gamma=0,5$ et la valeur numérique est obtenue avec la résolution de l'équation 16 pour différentes valeurs de $2 \gamma$. Les solutions obtenues ( Figure 1 ) pour $t=L / \alpha$ sont très voisines de la solution théorique calculée ( pour $\gamma=0,25$ ). La solution n'est donc que faiblement sensible à ces coefficients.

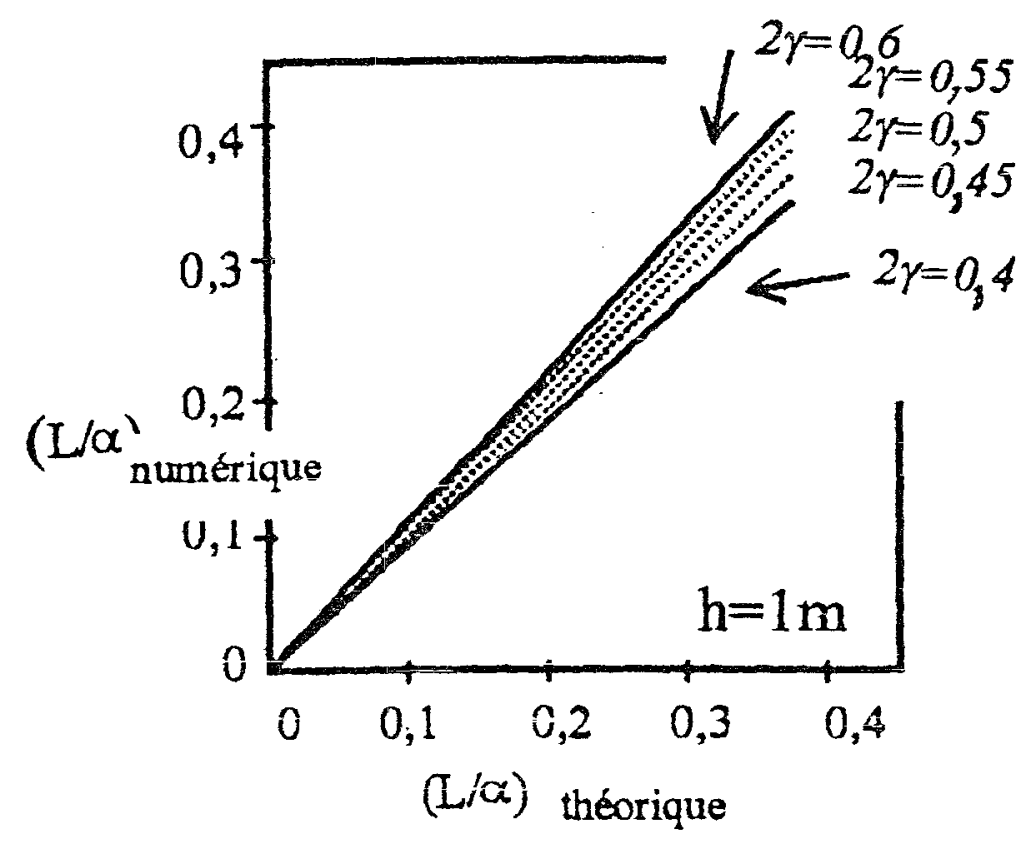

Fig.1.- Influence du coefficient $\gamma$ sur la longueur de mélange. Influence of $\gamma$ on the turbulent macroscale

Pour analyser l'influence des hypothèses effectuées, on utilise le modèie de turbulence $1 \mathrm{D}-\mathrm{V}$ basé sur la méthode K-L de Hyunh Thanh (1990). On calcule la longueur de mélange et la viscosité turbulente pour un écoulement de vitesse moyenne $1 \mathrm{~m} / \mathrm{s}$ sur une hauteur de $1 \mathrm{~m}$. et ou la rugosité de paroi est égale à 4,4 $10^{-2} \mathrm{~cm}$. Dans des conditions identiques au modèle analytique (gradient de pression nul ), les résultats sont similaires (avec $K$ maintenu constant ou non ).Les résultats sont présentés sur la figure 2 . La courbe $n^{\circ} 1$ correspond à la solution analytique linéaire et la courbe $\mathrm{n}^{\circ} 2$ à la nouvelle solution trouvée et présentée ici. La courbe $\mathrm{n}^{\circ} 3$ représente la solution numérique obtenue avec un gradient de 
pression nul et $\mathrm{K}$ variable. Par contre, si l'on résout numériquement le système avec $K$ variable et un gradient horizontal de pression non nul (système complet), nous constatons une variation du profil de la viscosité turbulente de $30 \%$ maximum, mais un changement moins important du profil de longueur de mélange. Le résultat est donné par la courbe $\mathrm{n}^{\circ} 4$. Ainsi la solution obtenue représente beaucoup mieux la réalité que la solution linéaire même en supprimant l'hypothèse de gradient de pression nul.
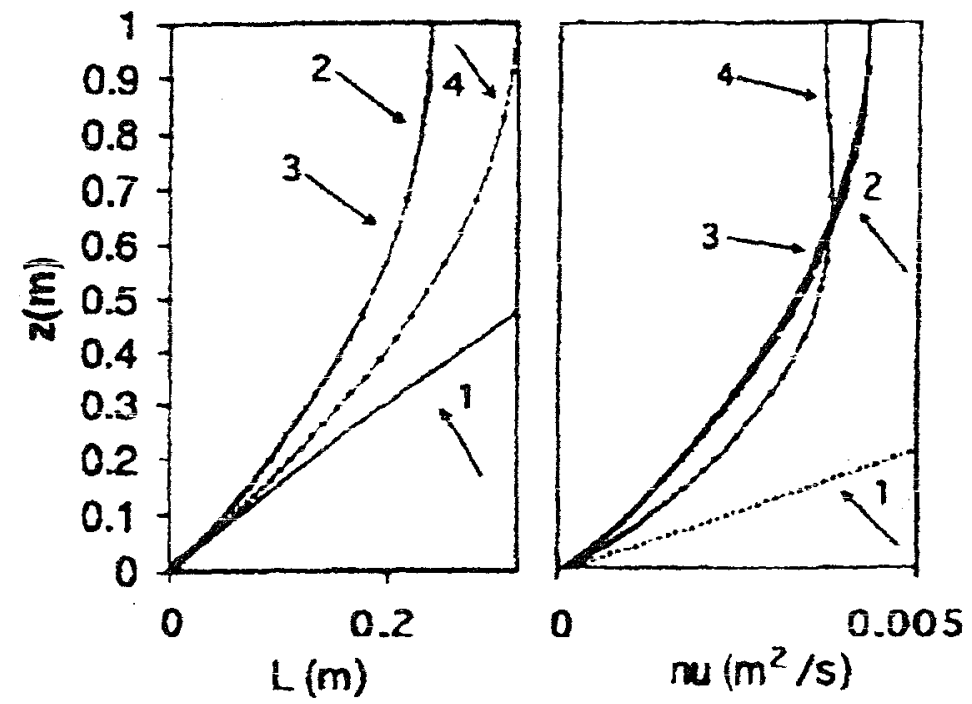

Fig. 2.- Influence des hypothèses sur la longueur de mélange $L$ et la viscosité turbulente $v_{i}$.

Influence of the hypothesis on the turbulent macroscale and turbulent viscosity

\section{Etude du profil des vitesses.}

Compte tenu que $V=2 \cos (\theta)$, on a vu au paragraphe 3 que $z=h[1+\cos (3 \theta)]$, et on en déduit que :

$$
d z=-\frac{3 h}{2}\left[1-V^{2}\right] d V
$$

Quand z croit de 0 à $h, \theta$ croit de $\pi / 3$ à $\pi / 2$ et $\mathrm{V}$ décroit de 1 à 0.

On sait aussi que $: \frac{d u}{d z}=\frac{\sqrt{K}}{L}=\frac{8 \sqrt{K}}{3 h \alpha\left(1-V^{2}\right)^{2}}$

En intégrant de la surface ( $V=0$ ) où la vitesse prend la valeur $u_{s}$ jusqu'à la hauteur $\mathrm{z}(\mathrm{ou} \mathrm{V})$, on obtient pour le profil des vitesses :

$u(z)=u_{s}+\int_{0}^{V} d u=u_{s}-\frac{4 \sqrt{K}}{\alpha} \int_{0}^{V} \frac{d V}{1-V^{2}}=u_{s}+\frac{2 \sqrt{K}}{\alpha} \operatorname{Ln}\left[\frac{1-V}{1+V}\right]$ 
Il est en général plus intéressant d'introduire la valeur $\bar{u}$ du courant moyenné sur la hauteur. On a :

$$
\begin{aligned}
& \bar{u}=\frac{1}{h} \int_{0}^{h} u(z) d z=u_{s}+\frac{3 \sqrt{K}}{\alpha} \int_{1}^{0}\left(1-V^{2}\right) \operatorname{Ln}\left[\frac{1-V}{1+V}\right] d V \\
& \bar{u}=u_{s}-\frac{2 \sqrt{K}}{\alpha} \ln \left(\frac{4}{\sqrt{e}}\right)
\end{aligned}
$$

On déduit le profil des vitesses :

$$
u(z)=\bar{u}++\frac{2 \sqrt{K}}{\alpha} \operatorname{Ln}\left(\frac{4}{\sqrt{e}} \frac{1-V}{1+V}\right)
$$

La valeur de la vitesse moyenne est atteinte à la cote $\bar{z}$ définie par :

$$
\frac{\bar{z}}{h}=1+\cos (3 \theta) \quad \text { avec } \quad \frac{1+2 \cos \theta}{1-2 \cos \theta}=\frac{4}{\sqrt{e}}
$$

On en déduit $\bar{z}=0,41158 \mathrm{~h}$

A titre de comparaison, pour la solution linéaire $L=\alpha z$, on a :

$\frac{d u}{d z}=\frac{\sqrt{K}}{\alpha z} \quad$ soit $\quad u=u_{s}+\frac{\sqrt{K}}{\alpha} \ln \frac{z}{h}=\bar{u}+\frac{\sqrt{K}}{\alpha} \ln \frac{z e}{h}$

La valeur moyenne de la vitesse est atteinte à la cote $\bar{z}$ :

$$
\bar{z}=\frac{h}{\varepsilon}=0,3678 h
$$

\section{Conclusion}

En conclusion, nous avons obtenu, dans le cas d'un écoulement permanent avec gradient de pression nul, une solution théorique pour l'échelle de longueur, plus réaliste que la solution linéaire qui correspond à un profil des vitesses logarithmique. Cefte solution permet d'obtenir une nouvelle expression analytique du profil des vitesses dans le cas d'un écoulement de Couette, et reste une bonne approximation pour le cas d'un gradient de pression non nul.

\section{Références bibliographiques}

CHEVALIER C., TEMPERVLLE A. (1999)- Etude analytique de la viscosité turbulente à l'aide du modèle $K-L, C$. R. Acd. Sci. Paris, t. 327, série II b,pp. 13711374. 
DAVIES A.G., SOULSBY R.L., KING H.L. (1988)- A mumerical model of the combined wave and current bottom boundary layer, J. Geophys. Res. 93, pp. 491508 .

GUIZIEN K., SILVA P. (1999)- Amélioration de la modélisation couplée houlecourant- sédiment, Colloque Génie Civil- Génie Côtier, Caen

HUYNH THANH S.,TEMPERVILLE A. (1990)- A numerical model of the rough turbulent boundary layer in combined wave and current interaction, Proc. of Int. Conf. in Coastal Eng., delft.

HUYNH THANH S., TRAN THU T., TEMPERVILLE A. (1990)-A numerical model for suspended sediment in combined currents and waves, Euromech 310, Le Havre, in Sediment Transport Mechanisms in Coastal Environments and Rivers, World Scientific, Singapore, pp.122-130.

LEWELLEN (1977) - Handbook of Turbulence, Vol. 1, W. Frost (Ed.), Plenum Publishing Corp., pp. 237-280. 